\title{
Growing Protean Graphs
}

\author{
Paweł Pratat and Nicholas Wormald
}

Abstract. The web may be viewed as a graph each of whose vertices corresponds to a static HTML web page and each of whose edges corresponds to a hyperlink from one web page to another. Recently, there has been considerable interest in using random graphs to model complex real-world networks to gain an insight into their properties. In this paper we propose an extended version of a new random model of the web graph in which the degree of a vertex depends on its age. We use the differential equation method to obtain basic results on the probability of edges being present. From this we are able to characterize the degree sequence of the model and study its behaviour near the connectivity threshold.

\section{Introduction}

Recently, many new random graphs models have been introduced and analyzed by certain common features observed in many large-scale, real-world networks such as the web graph (see, for instance, a general survey [Bonato 05]). The web may be viewed as a directed graph whose nodes correspond to static pages on the web and whose arcs correspond to links between these pages.

One of the most characteristic features of this graph is its degree sequence. Broder et al. [Broder et al. 00] noticed that the distribution of degrees follows a power law: the fraction of vertices with degree $d$ is proportional to $d^{-\gamma}$, where $\gamma$ is a constant independent of the size of the network (more precisely, $\gamma \sim 2.1$ for in-degrees, and $\gamma \sim 2.7$ for out-degrees). These observations suggest that the web is not well modeled by traditional random graph models such as $G_{n, p}$ (see, for instance, [Janson et al. 00]).

(C) A K Peters, Ltd. 
Euczak and the first author introduced another random graph model of the undirected web graphs, the protean graph $\mathcal{P}_{n}(d, \eta)$, which is controlled by two additional parameters $(d \in \mathbb{N}$ and $0<\eta<1)$ [Euczak and Prałat 06]. The major feature of this model is that older vertices are preferred when joining a new vertex into the graph. In the paper, it is proved that the degrees of the $\mathcal{P}_{n}(d, \eta)$ are distributed according to the power law. The first author also showed [Prałat 06] that the protean graph $\mathcal{P}_{n}(d, \eta)$ asymptotically almost surely (a.a.s.) has one giant component, containing a positive fraction of all vertices, whose diameter is equal to $\Theta(\log n)$.

Note that, unlike most of the theoretical models of the internet graph, the number of vertices of the protean graph is large but fixed and does not grow during the protean process. One may view this as a weakness of the approach since the internet graph is, at least at this moment, rapidly expanding. In the present paper, the authors introduce another random graph model, a growing protean graph $\mathcal{P}_{t}(p, d, \eta)$, which is an extended version of the standard protean graph controlled by an additional parameter $p, 0.5<p \leq 1$. This extension causes the number of vertices of $\mathcal{P}_{t}(p, d, \eta)$ to grow during the process.

In Section 4, we use the differential equation method to obtain a result (similar to [Euczak and Prałat 06, Lemma 3.5]) for the probability that a set of edges is present or absent in the graph, and then we use this result to derive degree distribution and connectivity properties of the growing protean graph, similar to those in the paper discussed above [Euczak and Prałat 06] (see Section 5).

\section{Definitions}

A protean process, defined below, is a sequence $\left\{G_{t}\right\}_{t=0}^{\infty}=\left\{\left(V_{t}, E_{t}\right)\right\}_{t=0}^{\infty}$ of undirected graphs, where $t$ denotes time. Our model has three fixed parameters: $0.5<p \leq 1, d \in N$, and $0<\eta<1$. Let $G_{0}=\left(V_{0}, E_{0}\right)=\left(\left\{v_{1}\right\}, \emptyset\right)$ be a fixed initial graph with a single vertex without edges. Let $N_{t}$ be a random variable denoting the number of vertices minus 1 at time $t$, i.e., $N_{t}=\left|V_{t}\right|-1$. For $t>0$, we form $G_{t}$ from $G_{t-1}$ according to the following rules:

- With probability $p$, add a new vertex $v=v_{N_{t-1}+1}$ together with $d$ edges from $v$ to existing vertices chosen randomly with weighted probabilities. The edges are added in $d$ substeps. In each substep, one edge is added, and the vertex to which to join is chosen as $v_{i}$ with probability equal to $i^{-\eta} / \sum_{j=1}^{N_{t-1}+1} j^{-\eta}$.

- Otherwise, which occurs with probability $1-p$, if $N_{t-1}=0\left(G_{t-1}\right.$ has a single vertex only) do nothing, whilst if $N_{t-1}>0$, choose a random vertex 
$v_{i}, i \in\left[N_{t-1}+1\right]=\left\{1,2, \ldots, N_{t-1}+1\right\}$, and delete $v_{i}$ together with all edges incident to it. Finally, relabel the remaining vertices preserving their order. Thus, $v_{j+1}$ becomes $v_{j}$ for $i \leq j \leq N_{t-1}$.

$\mathcal{P}_{t}(p, d, \eta)$ denotes the protean graph $G_{t}$.

Our model allows loops and multiple edges; there seems no reason to exclude them. However, there will not in general be very many of these, so excluding them can be shown not to significantly affect our conclusions.

There is also some flexibility in the starting graph. We could alternatively start with any arbitrary graph $G_{0}$, provided its vertices are assigned distinct "ages." Since all our results are asymptotic, it is easy to see that the same results will follow; the influence of the initial graph diminishes over time. In particular, our starting point in some proofs, such as the first result in Section 3, is the point at which the graph has grown suitably large but is otherwise arbitrary.

Note that during the process, a vertex $v_{j}$ "becomes" $v_{j-1}$. Since we want to track such changes for a particular vertex, we say that $v_{j}$ has label $j$ and regard the event of "becoming" $v_{j-1}$ as a change of label only. So, when this occurs, $v_{j}$ in $G_{t-1}$ is the same vertex as $v_{j-1}$ in $G_{t}$.

We say that an event holds with extreme probability (wep), if it holds with probability at least $1-\exp \left(-\Theta\left(\log ^{2} t\right)\right)$ as $t \rightarrow \infty$. More generally, an event holds wep $_{s}$ if it holds with probability at least $1-\exp \left(-\Theta\left(\log ^{2} s\right)\right)$ as $s \rightarrow \infty$. To combine this notion with other asymptotic notation, such as $O()$ and $o()$, we follow the conventions of the second author [Wormald 04].

\section{The Growing Protean Process}

We first show that the number of vertices of $G_{t}$ is concentrated.

Lemma 3.I. Let $p \in(0.5,1], d \in \mathbb{N}$, and $\eta \in(0,1)$. Then, wep

$$
N_{t}=N_{0}+(2 p-1) t+O(\sqrt{t} \log t)
$$

Proof. Let $\left\{Z_{i}\right\}$ be a sequence of $t$ independent random variables each of which is equal to 1 with probability $p$ and -1 with probability $1-p$. Then,

$$
N_{t}=N_{0}+\sum_{i=1}^{t} Z_{i}+f\left(N_{0},\left\{Z_{i}\right\}\right),
$$

where $f=f\left(N_{0},\left\{Z_{i}\right\}\right)$ is a deterministic function arising from the fact that a vertex is not deleted if $N_{i}$ is about to drop to zero. Since $f$ is nonnegative, the 
random variable $N_{t}$ is stochastically bounded from below by $N_{0}+\sum_{i=1}^{t} Z_{i}$. The lower tail of this variable has the sharp concentration, claimed by Chernoff's inequality (see, for instance, [Janson et al. 00, Corollary 2.3]). Thus, for every $\varepsilon=\Theta(\log t / \sqrt{t})$

$$
\begin{aligned}
\mathbb{P}\left(N_{t}<N_{0}\right. & +(1-\varepsilon)(2 p-1) t) \\
& \leq \mathbb{P}\left(\sum_{i=1}^{t} Z_{i}<(1-\varepsilon) \mathbb{E} \sum_{i=1}^{t} Z_{i}\right) \\
& \leq 2 \exp \left(-\frac{\varepsilon^{2}}{3} \mathbb{E} \sum_{i=1}^{t} Z_{i}\right)=\exp \left(-\Theta\left(\log ^{2} t\right)\right)
\end{aligned}
$$

For the upper tail, we note first (again using Chernoff's inequality) that wep the random variable $Z(k)=\sum_{i=1}^{k} Z_{i}$ is positive for every $k$ in the range $t^{1 / 4} \leq$ $k \leq t$. Hence, wep $f<t^{1 / 4}$. The upper tail bound again follows from Chernoff's inequality. For every $\varepsilon=\Theta(\log t / \sqrt{t})$,

$$
\begin{aligned}
\mathbb{P}\left(N_{t}>N_{0}\right. & +(1+\varepsilon)(2 p-1) t) \\
& =\mathbb{P}\left(\sum_{i=1}^{t} Z_{i}>(1+\varepsilon) \mathbb{E} \sum_{i=1}^{t} Z_{i}-f\right) \\
& \leq \mathbb{P}\left(\sum_{i=1}^{t} Z_{i}>\left(1+\frac{\varepsilon}{2}\right) \mathbb{E} \sum_{i=1}^{t} Z_{i}\right) \\
& \leq 2 \exp \left(-\frac{\varepsilon^{2}}{12} \mathbb{E} \sum_{i=1}^{t} Z_{i}\right)=\exp \left(-\Theta\left(\log ^{2} t\right)\right) .
\end{aligned}
$$

In the rest of this section, we will consider the growing protean process $\left\{G_{t}\right\}_{t=t_{0}}^{t_{f}}$ from a time $t_{0}$, conditional upon $G_{t_{0}}=G$ for some fixed graph $G$, and let $n$ denote the number of vertices of $G$ minus 1 , that is, $n=N_{t_{0}}=\left|V_{t_{0}}\right|-1$. For this section, we will consider the process only up to $t_{f}=t_{0}+\left\lfloor c n^{2 p} / \log ^{3} n\right\rfloor$, where $c>0$ is an arbitrary constant (included to make a nicer statement of Lemma 4.3). We desire effectively to assume that the vertex $v=v_{n+1} \in G_{t_{0}}$ survives until time $t_{f}$. Conditioning on this event, which we call $S\left(t_{0}, t_{f}\right)$, is equivalent to considering an altered process in which, for each step that deletes a vertex, the selection is made from the vertices other than $v$. Until further notice, we consider this altered process. We define the random variable $J_{t}$ to be the number of vertices older than $v$ in $G_{t}$. We need to show that $J_{t}$ is sharply 
concentrated in the context of the conditional space under consideration. It is easy for completeness to treat $N_{t}$ at the same time, even though Lemma 3.1 shows concentration of $N_{t}$ in general.

Note that the vector $\left(N_{t}, J_{t}\right)$ is Markovian, that is, its distribution at time $t+1$ is determined by its value at time $t$ and is independent of the earlier history. Also, it is easy to see that for every $t_{0} \leq t \leq t_{f}$, provided $N_{t}>0$,

$$
\begin{aligned}
\mathbb{E}\left(N_{t+1}-N_{t} \mid G_{t}\right) & =2 p-1, \\
\mathbb{E}\left(J_{t+1}-J_{t} \mid G_{t}\right) & =-(1-p) \frac{J_{t}}{N_{t}} .
\end{aligned}
$$

It provides some insight if we define real functions $z(x)$ and $y(x)$ to model the behaviour of the scaled functions $\frac{1}{n} N_{x n}$ and $\frac{1}{n} J_{x n}$, respectively. If we presume that the changes in the functions correspond to the expected changes of random variables, we obtain a system of differential equations

$$
\begin{aligned}
& z^{\prime}(x)=2 p-1, \\
& y^{\prime}(x)=-(1-p) \frac{y}{z},
\end{aligned}
$$

with the initial conditions $z\left(t_{0} / n\right)=y\left(t_{0} / n\right)=1$. The general solution of this system can be put in the form

$$
\begin{aligned}
(2 p-1) \log y & =-(1-p) \log z+C_{1} \\
z & =(2 p-1) x+C_{2} .
\end{aligned}
$$

Defining

$$
H\left(N_{t}, J_{t}\right)=(2 p-1) \log \frac{J_{t}}{n}+(1-p) \log \frac{N_{t}}{n},
$$

in view of (3.1), the general solution of the scaled differential equation corresponds to the system of equations

$$
\begin{aligned}
H\left(N_{t}, J_{t}\right) & =C_{1}, \\
N_{t}-(2 p-1) t & =C_{2} .
\end{aligned}
$$

This is a solution (taking $N_{t}=N(t)$ etc.) of the unscaled differential equations

$$
\begin{aligned}
N^{\prime}(t) & =2 p-1, \\
J^{\prime}(t) & =-(1-p) \frac{J}{N},
\end{aligned}
$$

where $t$ is regarded as a real variable. Of course, $C_{1}$ and $C_{2}$ are determined by the initial conditions. It should be emphasised that these differential equations are only suggested (at this stage). However, we will be able to show that $J_{t}$ is well concentrated around the solution value $y(t / n) n$. For this we use the same supermartingale method used by Pittel et al. [Pittel et al. 96]. It is encapsulated by the following result [Wormald 99, Corollary 4.1]. 
Lemma 3.2. Let $G_{0}, G_{1}, \ldots, G_{t}$ be a random process and $X_{i}$ a random variable determined by $G_{0}, G_{1}, \ldots, G_{i}, 0 \leq i \leq t$. Suppose that for some real $b$ and constants $c_{i}, \mathbb{E}\left(X_{i}-X_{i-1} \mid G_{0}, G_{1}, \ldots, G_{i-1}\right)<b$ and $\left|X_{i}-X_{i-1}-b\right| \leq c_{i}$ for $1 \leq i \leq t$. Then, for all $\alpha>0$,

$$
\mathbb{P}\left(\exists i(0 \leq i \leq t): X_{i}-X_{0} \geq i b+\alpha\right) \leq \exp \left(-\frac{\alpha^{2}}{2 \sum c_{j}^{2}}\right)
$$

We now come to the main result of this section. Let $I_{t_{0}, i, t}$ denote the label of the vertex in $G_{t}$ that was $v_{i}$ in the graph $G_{t_{0}}$, provided that vertex is still present in $G_{t}$. Note that we can express $J_{t}$ in terms of this notation, that is, $J_{t}=I_{t_{0}, N_{t_{0}}+1, t}-1$. When $i$ and $t_{0}$ are understood, we abbreviate this to $I_{t}$.

Theorem 3.3. Let $p \in(0.5,1], d \in \mathbb{N}$, and $\eta \in(0,1)$; for arbitrary $t_{0}$ let $G_{t}, N_{t}$, and $J_{t}$ be defined as above; and let $i \leq N_{t_{0}}$. Condition on the events that $G_{t_{0}}=G$ for some fixed graph $G$ and that $S\left(t_{0}, t_{f}\right)$ holds, and put $n=N_{t_{0}}=|V(G)|-1$. Then, wep $p_{n}$, for every $t$ in the range $t_{0} \leq t \leq t_{f}$, we have

$$
\begin{aligned}
& N_{t}=n+(2 p-1)\left(t-t_{0}\right)+O\left(n^{p} \log n\right), \\
& J_{t}=n\left(\frac{N_{t}}{n}\right)^{\frac{p-1}{2 p-1}}\left(1+O\left(\log ^{-1 / 2} n\right)\right),
\end{aligned}
$$

and, conditional upon the vertex $v_{i} \in G_{t_{0}}$ surviving until time $t_{f}$,

$$
I_{t}=\frac{i J_{t}}{n}\left(1+O\left(\log ^{-1 / 2} n\right)\right) \text { or } \frac{i J_{t}}{n}<\log ^{3} n .
$$

Proof. In the first main part of the proof, we show (3.5), and with almost no effort we obtain (3.4) at the same time. Alternatively, one can at the outset obtain (3.4) as follows. Note that for every $t_{0} \leq t \leq t_{0}+n^{p}$ Equation (3.4) holds (deterministically). We observe that Lemma 3.1 applies for the growing protean process starting from an arbitrary initial graph with $N_{0}+1$ vertices. This implies immediately that wep $_{t-t_{0}}$

$$
N_{t}=n+(2 p-1)\left(t-t_{0}\right)+O\left(\sqrt{t-t_{0}} \log \left(t-t_{0}\right)\right)
$$

holds for every $t_{0} \leq t \leq t_{f}$. So, wep $n$ for every $t_{0}+n^{p}<t \leq t_{f}$, (3.4) holds.

Let $\mathbf{w}_{t}=\left(N_{t}, J_{t}\right)$, and consider the sequence of random variables

$$
\left\{X_{t}\right\}_{t=t_{0}}^{t_{f}}=\left\{H\left(\mathbf{w}_{t}\right)\right\}_{t=t_{0}}^{t_{f}},
$$


where the function $H$ is defined in (3.2) and the stopping time is

$$
T=\min \left\{t \geq t_{0}: J_{t}<n^{p} / 2 \vee N_{t}<n / 2 \vee t=t_{f}\right\} .
$$

(A stopping time is any random variable $T$ with values in $\{0,1, \ldots\} \cup\{\infty\}$ such that it is determined whether $T=\hat{t}$ for any time $\hat{t}$ from knowledge of the process up to and including time $\hat{t}$.) Note that the second-order partial derivatives of $H$ with respect to $N_{t}$ and $J_{t}$ are $O\left(1 / N_{t}^{2}+1 / J_{t}^{2}\right)=O\left(1 / J_{t}^{2}\right)=O\left(n^{-2 p}\right)$, provided $T>t$. Therefore, with $i \wedge T$ denoting $\min \{i, T\}$, we have

$$
\begin{aligned}
& H\left(\mathbf{w}_{(t+1) \wedge T}\right)-H\left(\mathbf{w}_{t \wedge T}\right) \\
& \quad=\left(\mathbf{w}_{(t+1) \wedge T}-\mathbf{w}_{t \wedge T}\right) \cdot \operatorname{grad} H\left(\mathbf{w}_{t \wedge T}\right)+O\left(n^{-2 p}\right) .
\end{aligned}
$$

Recall that $H(\mathbf{w})$ is constant along every trajectory $\mathbf{w}$ of the unscaled differential equations (3.3). So, taking the expectation of (3.7) conditional on $G_{t \wedge T}$, we obtain

$$
\mathbb{E}\left(H\left(\mathbf{w}_{(t+1) \wedge T}\right)-H\left(\mathbf{w}_{t \wedge T}\right) \mid G_{t \wedge T}\right)=O\left(n^{-2 p}\right) .
$$

Also, from (3.7), noting that

$$
\operatorname{grad} H\left(\mathbf{w}_{t}\right)=\left(O\left(1 / N_{t}\right), O\left(1 / J_{t}\right)\right),
$$

and from the fact that $N_{t}$ and $J_{t}$ change by at most 1 per each step of the process, we also have

$$
\begin{aligned}
& \left|H\left(\mathbf{w}_{(t+1) \wedge T}\right)-H\left(\mathbf{w}_{t \wedge T}\right)\right| \\
& \quad=O\left(1 / N_{t \wedge T}\right)+O\left(1 / J_{t \wedge T}\right)+O\left(n^{-2 p}\right)=O\left(n^{-p}\right) .
\end{aligned}
$$

Now we can apply Lemma 3.2 to the sequence $\left\{H\left(\mathbf{w}_{t \wedge T}\right)\right\}_{t=t_{0}}^{t_{f}}$, and symmetrically to $\left\{-H\left(\mathbf{w}_{t \wedge T}\right)\right\}_{t=t_{0}}^{t_{f}}$, with $\alpha=\log ^{-1 / 2} n, b=O\left(n^{-2 p}\right)$ and $c_{j}=O\left(n^{-p}\right)$, to show that $\operatorname{wep}_{n}$

$$
\left|H\left(\mathbf{w}_{t \wedge T}\right)-H\left(\mathbf{w}_{t_{0}}\right)\right|=O\left(\log ^{-1 / 2} n\right) .
$$

As $H\left(\mathbf{w}_{t_{0}}\right)=0$, this implies from the definition (3.2) of the function $H$, that wep $_{n}$ Equation (3.5) holds for every $t_{0} \leq t \leq T$. By the same type of argument, but much simpler, we immediately obtain (3.4) $\mathrm{wep}_{n}$ for $t$ up to $T$.

To complete the first part of the proof, we need to show that wep $n, T=t_{f}$. The events asserted by (3.4) and (3.5) hold with this probability up until time $T$, as shown above, and the conjunction of these events implies that $J_{t}>n^{p} / 2$ and $N_{t}>n / 2$ for $n$ sufficiently large, $t_{0} \leq t \leq T$. It follows that $T=t_{f} \operatorname{wep}_{n}$. Together with the conclusion above, this completes the proof of the claim on the distribution of $N_{t}$ and $J_{t}$. 
We now turn to the claim on the distribution of the random variable $I_{t}$. It is easy to observe that, conditional upon $v_{i}$ surviving until time $t_{f}, I_{t}$ follows the hypergeometric distribution with parameters $n-2, k-1$, and $J_{t}-2$, that is, conditional upon it surviving,

$$
\mathbb{P}\left(I_{t}=k \mid J_{t}\right)=\frac{\left(\begin{array}{c}
i-1 \\
k-1
\end{array}\right)\left(\begin{array}{c}
n-i-1 \\
J_{t}-k-1
\end{array}\right)}{\left(\begin{array}{c}
n-2 \\
J_{t}-2
\end{array}\right)}
$$

Thus,

$$
\mathbb{E}\left(I_{t} \mid J_{t}\right)=\frac{(i-1)\left(J_{t}-2\right)}{n-2}=\frac{i J_{t}}{n}\left(1+O\left(n^{p-1}\right)\right) .
$$

We can apply a well-known bound for the tail of the hypergeometric distribution (see, for instance, [Janson et al. 00, Theorem 2.10]) to show that the random variable $I_{t}$ is sharply concentrated around its mean. Indeed, working in the conditional space under consideration (that the vertex survives and that $J_{t}$ is given) using the fact that $\mathbb{E} I_{t} \geq \log ^{3} n$, we obtain

$$
\mathbb{P}\left(\left|I_{t}-\mathbb{E} I_{t}\right|>\frac{\mathbb{E} I_{t}}{\log ^{1 / 2} n}\right) \leq 2 \exp \left(-\frac{\mathbb{E} I_{t}}{3 \log n}\right)=\exp \left(-\Omega\left(\log ^{2} n\right)\right),
$$

which is the assertion required for (3.6).

It is straightforward to obtain results like those in Theorem 3.3 but with much smaller error bounds than $O\left(\log ^{-1 / 2} n\right)$, at the expense of reducing the value of $t_{f}$. One could then apply the lemma to successive intervals of time, tracking the progress of vertices in the later intervals using (3.6). However, to obtain the main corollaries in later sections, this is not required. We do, however, need to convert the theorem to a form that does not require conditioning on $G_{t_{0}}$, as follows.

Corollary 3.4. Let $p \in(0.5,1], d \in \mathbb{N}$, and $\eta \in(0,1)$. For arbitrary $t_{0}$ define $J_{t}$ and $I_{t}$ as above, and define $t_{F}=t_{0}+\left\lfloor c t_{0}^{2 p} / \log ^{3} t_{0}\right\rfloor$, where $c>0$ is an arbitrary constant. Let $D\left(i, t_{0}, t\right)$ denote the event that either $i>N_{t_{0}}+1$ or the vertex of label $i$ in $G_{t_{0}}$ is not still present in $G_{t}$. Then, wep $t_{0}$ for every $t$ in the range $t_{0} \leq t \leq t_{F}$, we have

$$
\begin{aligned}
N_{t} & =(2 p-1) t+O\left(t_{0}^{p} \log t_{0}\right), \\
D\left(N_{t_{0}}+1, t_{0}, t\right) \quad \text { or } J_{t} & =N_{t_{0}}\left(\frac{N_{t}}{N_{t_{0}}}\right)^{\frac{p-1}{2 p-1}}\left(1+O\left(\log ^{-1 / 2} t_{0}\right)\right),
\end{aligned}
$$

and for all $i>0$

$$
D\left(i, t_{0}, t\right) \text { or } I_{t}=\frac{i J_{t}}{N_{t_{0}}}\left(1+O\left(\log ^{-1 / 2} t_{0}\right)\right) \text { or } \frac{i J_{t}}{N_{t_{0}}}<\log ^{3} t_{0} .
$$


Proof. Lemma 3.1 shows that $N_{t_{0}}=\Theta\left(t_{0}\right)$ wep $_{t_{0}}$. Then, conditioning on the event that $N_{t_{0}}=\Theta\left(t_{0}\right),(3.4)$ implies that (3.8) holds wep $t_{0}$. It then holds wep $t_{0}$ without the conditioning since $N_{t_{0}}=\Theta\left(t_{0}\right)$ wep $_{t_{0}}$. We obtain (3.9) and (3.10) similarly; replacing the conditioning in Theorem 3.3 by the disjunction with the event $D\left(i, t_{0}, t\right)$ merely weakens the result.

\section{Basic Lemma for Edge Probabilities}

In this section we introduce the main tool that allows us easily to compute the probability of some events in the protean graphs, Lemma 4.3. This shows a relationship between $\mathcal{P}_{t}(p, d, \eta)$ and the random graph $G(n, q)$ on the set of vertices $[n]=\{1,2, \ldots, n\}$ (where $n=N_{t}+1$ ), in which a pair of vertices $i, j$, $1 \leq i<j \leq n$, are adjacent with probability

$$
q=q(i, j)=d(1-\eta) n^{(p-1) / p} j^{\eta+(1-2 p) / p} i^{-\eta}
$$

independently for each such pair. Of course, the protean graph $\mathcal{P}_{t}(p, d, \eta)$ has a very rich dependence structure, so it only shares some properties with $G(n, q)$.

First, we consider a generalization of the well-known balls-into-bins model, which will be useful to prove Lemma 4.3. Suppose that we sequentially put $d$ balls into $m$ bins by placing each ball into a bin independently, and the probability that we choose a bin $k, 1 \leq k \leq m$, is equal to $\rho_{k}$, where $\sum_{i=1}^{m} \rho_{k}=1$. Let $S_{1}, S_{2} \subseteq[m], S_{1} \cap S_{2}=\emptyset,\left|S_{1}\right| \leq d$, and $p\left(S_{1}, S_{2}\right)$ denote the probability that every bin from the set $S_{1}$ has at least one ball and bins from the set $S_{2}$ have no balls. In the following, we use the notation $[x]_{k}=x(x-1) \cdots(x-k+1)$.

The following fact was used in [Euczak and Prałat 06].

Fact 4.I. Using the notation above, we have

$$
p\left(S_{1}, S_{2}\right) \geq\left(1-\sum_{j \in S_{1} \cup S_{2}} \rho_{j}\right)^{d-\left|S_{1}\right|}[d]_{\left|S_{1}\right|} \prod_{i \in S_{1}} \rho_{i}
$$

and

$$
p\left(S_{1}, S_{2}\right) \leq\left(1-\sum_{j \in S_{2}} \rho_{j}\right)^{d-\left|S_{1}\right|}[d]_{\left|S_{1}\right|} \prod_{i \in S_{1}} \rho_{i} .
$$

The proof is simple: in the first inequality $p\left(S_{1}, S_{2}\right)$ is estimated by the probability that each bin from $S_{1}$ contains precisely one ball; in the second, some configurations are counted more than once. 
Note that we may consider the process as two separate processes. The first process adds and deletes vertices and decides what the vertex sets are for all graphs $G_{t}$. Let us call this the vertex process. The second process (edge process) then decides which pairs of vertices are adjacent by using the rules of the growing protean process, at each time $t$, for the vertex added. We will consider the vertex process first, and when we have enough facts about it at our fingertips, we will consider the edge process. Note that Corollary 3.4 only really describes the vertex process.

Before stating the main results of this section, we define $n=n(t)$ to be the deterministic function of $t$ that approximates the number of vertices in $\mathcal{P}_{t}(p, d, \eta)$; that is,

$$
n=n(t)=(2 p-1) t
$$

Given $n, p, d$, and $\eta$, define

$$
u(j)=j^{(2 p-1) / p} n^{(1-p) / p}
$$

and

$$
w(i, j)=(1-\eta)(j / i)^{\eta} / u(j)=(1-\eta) n^{(p-1) / p} j^{\eta+(1-2 p) / p} i^{-\eta} .
$$

Note that

$$
w(i, j)=\left(1+O\left(u(j)^{\eta-1}\right)\right) \frac{(i u(j) / j)^{-\eta}}{\sum_{s=1}^{u(j)} s^{-\eta}} .
$$

First, we need to "invert" Corollary 3.4 to obtain a statement that gives information about the vertex process for many times $t_{0}$ earlier than $t$.

Lemma 4.2. Let $j_{0}=\sqrt{t} \log ^{3 /(4 p-2)} t$. Then, wep for every $i$ and $j$ with $2 \log ^{3} t<$ $i<j \leq N_{t}+1$ and $j>j_{0}$, the vertex with label $j$ at time $t$ was added at time

$$
\hat{t}=\frac{j^{(2 p-1) / p} N_{t}^{(1-p) / p}}{2 p-1}\left(1+O\left(\log ^{-1 / 2} t\right)\right) .
$$

Furthermore, if we let $\hat{i}$ denote the label in $G_{\hat{t}}$ of the vertex of label $i$ in $G_{t}$, then wep

$$
\hat{i}=\frac{i N_{\hat{t}}}{j}\left(1+O\left(\log ^{-1 / 2} t\right)\right)=\frac{i u(j)}{j}\left(1+O\left(\log ^{-1 / 2} t\right)\right) .
$$

Proof. Put $t_{I}=c^{\prime} t^{1 /(2 p)} \log ^{3 /(2 p)} t$ for some $c^{\prime}>0$. Then, for $t_{I} \leq t_{0} \leq t$, wep $t_{0}$ is equivalent to wep. Also, for any such $t_{0}$, we may apply Corollary 3.4 since, for appropriate $c$ in that corollary, $t<t_{F}$. Since a polynomial number of statements holding individually wep also hold jointly wep, we deduce that wep Equations 
(3.8), (3.9), and (3.10) hold simultaneously for all $t_{0}$ in the range $t_{I} \leq t_{0} \leq t$. By the same argument and Lemma 3.1, we have that wep

$$
N_{t_{0}}=(2 p-1) t_{0}+O\left(\sqrt{t_{0}} \log t_{0}\right)
$$

for all $t_{0}$ in the same range.

For some fixed $C>0$, define $t_{1}=\left\lfloor t_{1}^{*}\right\rfloor$ where

$$
t_{1}^{*}=\frac{j^{(2 p-1) / p} N_{t}^{(1-p) / p}\left(1-C \log ^{-1 / 2} t\right)}{2 p-1} .
$$

In view of the conclusions above, the following statements hold wep. From (4.2), for every $t_{3}, t_{I} \leq t_{3} \leq t_{1}$,

$$
\begin{aligned}
N_{t_{3}} & =(2 p-1) t_{3}\left(1+O\left(t_{3}^{-1 / 2} \log t_{3}\right)\right) \\
& \leq(2 p-1) t_{1}^{*}\left(1+O\left(t_{3}^{-1 / 2} \log t_{3}\right)\right) \\
& =j^{(2 p-1) / p} N_{t}^{(1-p) / p}\left(1-C \log ^{-1 / 2} t\right)\left(1+O\left(t_{I}^{-1 / 2} \log t_{I}\right)\right) \\
& \leq j^{(2 p-1) / p} N_{t}^{(1-p) / p}\left(1-c^{\prime \prime} C \log ^{-1 / 2} t_{I}\right) \\
& \leq j^{(2 p-1) / p} N_{t}^{(1-p) / p}\left(1-c^{\prime \prime} C \log ^{-1 / 2} t_{3}\right)
\end{aligned}
$$

where $c^{\prime \prime}>0$ is a constant, since $t=\Theta\left(t_{I}^{2 p} / \log ^{3} t_{I}\right)$. Now, from the statement above using (3.9), for sufficiently large $C$ all vertices added at any time in the interval $\left[t_{I}, t_{1}\right]$ have label strictly less than $j$ if they survive until time $t$. This statement holds wep.

Note that, for small enough $c^{\prime}, t_{I}$ can be made an arbitrarily small fraction of $t_{1}$. So for any time $\hat{t}, t^{1 / 2}<\hat{t}<t_{I}$, we may apply the same argument except we reduce the value of $t$ to a smaller value, $t^{\prime}$ - and if convenient reduce $j$ to a smaller value, $j^{\prime}$ - to deduce that wep the vertex added at time $\hat{t}$ has label strictly less than $j$ at time $t^{\prime}$, if it survives until then. Since the label of a vertex cannot increase as the process continues, this is also true at time $t$. We may thus extend the interval to encompass all vertices added in the time interval $\left[t^{1 / 2}, t_{1}\right]$. Of course, vertices added before this interval have label less than $t^{1 / 2}<j_{0} \leq j$. So, for sufficiently large $C$, wep all vertices added at any time before $t_{1}$ have label strictly less than $j$ if they survive until time $t$.

Similarly, if we define $t_{2}=\left\lceil t_{2}^{*}\right\rceil$ where

$$
t_{2}^{*}=\frac{j^{(2 p-1) / p} N_{t}^{(1-p) / p}\left(1+C \log ^{-1 / 2} t\right)}{2 p-1},
$$


then wep all vertices added at any time in the interval $\left[t_{2}, t\right]$ have label strictly greater than $j$ at time $t$, if they survive until then.

We deduce from these conclusions that wep for all $j \in\left[j_{0}, N_{t}\right]$, the vertex with label $j$ at time $t$ was added at some time between $t_{1}=t_{1}(j)$ and $t_{2}=t_{2}(j)$. In view of Corollary 3.4, we may approximate $N_{t}$ by $n$, and this gives the first statement in the lemma.

Again from the above observations, the number of vertices at such a time $\hat{t}$, $t_{1} \leq \hat{t} \leq t_{2}$, is wep equal to (recalling the definitions (4.1), etc.)

$$
\begin{aligned}
N_{\hat{t}} & =\hat{t}(2 p-1)\left(1+O\left(\hat{t}^{-1 / 2} \log \hat{t}\right)\right) \\
& =j^{(2 p-1) / p} n^{(1-p) / p}\left(1+O\left(\log ^{-1 / 2} t\right)\right) \\
& =u(j)\left(1+O\left(\log ^{-1 / 2} t\right)\right) .
\end{aligned}
$$

Taking $t_{0} \in\left[t_{1}, t_{2}\right]$, as mentioned above, we may assume that (3.10) holds wep. Hence, using a sandwiching argument as above (but for positions rather than times), if we let $\hat{i}$ denote the label in $G_{t_{0}}$ of the vertex of label $i$ in $G_{t}$, then wep

$$
\hat{i}=\frac{i N_{t_{0}}}{j}\left(1+O\left(\log ^{-1 / 2} t\right)\right)=\frac{i u(j)}{j}\left(1+O\left(\log ^{-1 / 2} t\right)\right)
$$

(Note in particular that the condition $2 \log ^{3} t \leq i$ ensures that the condition $\frac{\hat{i} J_{t}}{N_{t_{0}}}<\log ^{3} t_{0}$ wep does not hold.) This gives the second assertion of the lemma.

We will use the following lemma to estimate the probability that pairs of vertices are adjacent in $G_{t}$ and that others are not.

Lemma 4.3. Let $0.5<p \leq 1, d \in \mathbb{N}, 0<\eta<1$,

$$
E_{1}, E_{2} \subseteq\left\{\left\{v_{i}, v_{j}\right\}: 2 \log ^{3} t<i<j \leq N_{t}+1 \text { and } j \geq j_{0}\right\} \text {, and } E_{1} \cap E_{2}=\emptyset \text {. }
$$

For every $i, j \in\left[N_{t}+1\right]$ and $r=1,2$, let

$$
\begin{gathered}
V_{r}(j)=\left\{i: i<j \text { and }\left\{v_{i}, v_{j}\right\} \in E_{r}\right\}, \\
w_{r}(j)=\sum_{i \in V_{r}(j)} w(i, j),
\end{gathered}
$$

and assume that $\left|V_{1}(j)\right| \leq d$ for every $j \in[n]$. 
Let $P_{t}\left(E_{1}, E_{2}, p, d, \eta\right)$ denote the probability that all pairs from $E_{1}$ are edges of $\mathcal{P}_{t}(p, d, \eta)$ and no pair from $E_{2}$ is an edge of $\mathcal{P}_{t}(p, d, \eta)$. Then,

$$
\begin{aligned}
P_{t}\left(E_{1}, E_{2}, p, d, \eta\right) \leq & o\left(\exp \left(-\log ^{3 / 2} t\right)\right) \\
& +\prod_{j=1}^{n}\left[1-\left(1+O\left(\log ^{-1 / 2} t\right)\right) w_{2}(j)\right]^{d-\left|V_{1}(j)\right|} \\
& \times[d]_{\left|V_{1}(j)\right|} \prod_{i \in V_{1}(j)}\left(1+O\left(\log ^{-1 / 2} t\right)\right) w(i, j),
\end{aligned}
$$

and

$$
\begin{aligned}
P_{t}\left(E_{1}, E_{2}, p, d, \eta\right) \geq & o\left(\exp \left(-\log ^{3 / 2} t\right)\right) \\
& +\prod_{j=1}^{n}\left[1-\left(1+O\left(\log ^{-1 / 2} t\right)\right)\left(w_{1}(j)+w_{2}(j)\right)\right]^{d-\left|V_{1}(j)\right|} \\
& \times[d]_{\left|V_{1}(j)\right|} \prod_{i \in V_{1}(j)}\left(1+O\left(\log ^{-1 / 2} t\right)\right) w(i, j)
\end{aligned}
$$

Proof. For this we need to consider the edge process as defined at the start of this section. To obtain conclusions wep, we may condition on any of the times $t_{0}$ that vertex of label $j$ in $G_{t}$ was added in the vertex process, with $t_{1}(j) \leq t_{0} \leq t_{2}(j)$. The assertion then follows from Fact 4.1, Lemma 4.2, and the definition of the edge process.

From Lemma 4.3 it follows that the behaviour of the protean graph $\mathcal{P}_{t}(p, d, \eta)$ is related to that of the random graph with vertex set $[n]$ in which two vertices $i$ and $j, 2 \log ^{3} t \leq i<j \leq n, j_{0} \leq j$, are adjacent with probability

$$
p(i, j)=d w(i, j)=d(1-\eta) n^{(p-1) / p} j^{\eta+(1-2 p) / p} i^{-\eta},
$$

independently for each such pair.

Indeed, if $\left|V_{1}(j)\right|=o(d)$ for every $j \in[n]$, then Lemma 4.3 gives

$$
\begin{aligned}
P_{t}\left(E_{1}, E_{2}, p, d, \eta\right) & \sim \prod_{j=1}^{n}\left(1-\sum_{i \in V_{2}(j)} w(i, j)\right)^{d} d^{\left|V_{1}(j)\right|} \prod_{i \in V_{1}(j)} w(i, j) \\
& =(1+o(1)) \exp \left(-\sum_{\{i, j\} \in E_{2}} p(i, j)\right) \prod_{\{i, j\} \in E_{1}} p(i, j),
\end{aligned}
$$

whereas if we consider a graph with independent edges, the probability that an 
analogous event holds is equal to

$$
\begin{aligned}
\prod_{\{i, j\} \in E_{2}}(1-p(i, j)) & \prod_{\{i, j\} \in E_{1}} p(i, j) \\
& =(1+o(1)) \exp \left(-\sum_{\{i, j\} \in E_{2}} p(i, j)\right) \prod_{\{i, j\} \in E_{1}} p(i, j) .
\end{aligned}
$$

Finally, note that the situation with these results is similar to that in [Euczak and Prałat 06]: since we claim nothing about edges between 'small' vertices $i$, $1 \leq i<2 \log ^{3} t$, it is difficult to obtain a general theorem which relates properties of our model to the model with independent edges (as is done, for instance, for a different model by Chung and $\mathrm{Lu}$ [Chung and $\mathrm{Lu} \mathrm{04]).} \mathrm{For} \mathrm{the} \mathrm{same} \mathrm{reason}$ we cannot use the general theory of Bollobás, Janson and Riordan [Bollobás et al. 05] of inhomogeneous sparse random graphs. Nonetheless, as in [Łuczak and Prałat 06], our Lemma 4.3 is strong enough to show that many properties of the independent model which, roughly speaking, do not depend on the behaviour of the first $2 \log ^{3} t$ vertices, hold also for the growing protean graph. We discuss some examples in the next section.

\section{Degrees of Vertices and Connectivity}

In this section we study the shape of the degree sequence of $\mathcal{P}_{t}(p, d, \eta)$ and its connectivity. The proofs are virtually the same as those for the corresponding results in [Euczak and Prałat 06], but with our new Lemma 4.3 in place of Lemma 3.5 of that paper. We begin with the expected degree of vertex $v_{i}$. Recall that $j_{0}=j_{0}(t)=\sqrt{t} \log ^{3 /(4 p-2)} t$ and $n=n(t)=(2 p-1) t$.

Theorem 5.I. Let $0.5<p \leq 1, d=o\left(t^{(1-\eta) / 3}\right)$, and $0<\eta<1$. Then, the expected degree of a vertex $v_{i=i(t)}$ is given by

$$
\mathbb{E} d\left(v_{i}\right) \sim d \frac{1-\eta}{(1-p) / p+\eta}\left(\left(\frac{n}{i}\right)^{\eta}+\frac{(1-2 p) / p+2 \eta}{1-\eta}\left(\frac{i}{n}\right)^{(1-p) / p}\right)
$$

for $j_{0}<i \leq N_{t}+1$ and

$$
\mathbb{E} d\left(v_{i}\right) \sim d \frac{1-\eta}{(1-p) / p+\eta}\left(\frac{n}{i}\right)^{\eta}
$$

for $2 \log ^{3} t<i \leq j_{0}$. Moreover, the expected number of edges in the protean graph $\mathcal{P}_{t}(p, d, \eta)$ is equal to $(1+o(1)) p d n$. 
Note that for small $i=o(n)$, the expected degree of the vertex $v_{i}$ is dominated by the factor $d \frac{1-\eta}{(1-p) / p+\eta}\left(\frac{n}{i}\right)^{\eta}$. Consequently, the degrees of the protean graph $\mathcal{P}_{t}(p, d, \eta)$ are distributed according to a power law. More specifically, let $Z_{k}=$ $Z_{k}(n ; p ; d ; \eta)$ denote the number of vertices of degree $k$ in $\mathcal{P}_{t}(p, d, \eta)$ and $Z_{\geq k}=$ $\sum_{\ell \geq k} Z_{\ell}$. Here and below a.a.s. means "with probability tending to 1 as $n \rightarrow \infty$."

Theorem 5.2. Let $0.5<p \leq 1, d \in \mathbb{N}, 0<\eta<1, k=k(n) \geq \log ^{2} n$, and $d=o(k)$. Then, a.a.s.

$$
Z_{\geq k}=(1+o(1)) n\left(\frac{1-\eta}{(1-p) / p+\eta} \cdot \frac{d}{k}\right)^{1 / \eta}+O\left(\log ^{3} n\right) .
$$

As with the nongrowing protean graph in [Łuczak and Prałat 06], we may attune the parameters of this model to obtain roughly the same degree distribution as the (undirected) web graph.

We next consider the connectivity of $\mathcal{P}_{t}(p, d, \eta)$. Let $\rho_{t}(p, d, \eta)$ denote the probability that $\mathcal{P}_{t}(p, d, \eta)$ is connected.

Theorem 5.3. Let $0.5<p \leq 1,0<\eta<1$, and $d=d(n)=a \log n$, where $a$ is a positive constant. Then,

$$
\lim _{t \rightarrow \infty} \rho_{t}(p, d, \eta)= \begin{cases}1 & \text { if } \quad a>1 / g\left(x_{0}\right) \\ 0 & \text { if } \quad a<1 / g\left(x_{0}\right)\end{cases}
$$

where

$$
g(x)=\frac{1-\eta}{(1-p) / p+\eta}\left(x^{-\eta}-x^{(1-p) / p}\right)-\log \left(1-x^{(1-p) / p}\right)
$$

and $x_{0}=x_{0}(p, \eta)$ is a value of $x$ that minimizes function $g(x)$ in the interval $(0,1)$.

We observe that, as for the model in [Euczak and Prałat 06], a.a.s. near the threshold all isolated vertices have labels $(1+o(1)) x_{0}(p, \eta) n$. The probability of being isolated is greatest for the vertices of medium labels since they have lost their old neighbours that have already been deleted, yet they are not old enough to attract new ones.

Acknowledgements. The second author was supported by the Canada Research Chairs Program and NSERC. 


\section{References}

[Bollobás et al. 05] B. Bollobás, S. Janson, and O. Riordan. "The Phase Transition in Inhomogeneous Random Graphs." Technical Report 2005:18, Uppsala, 2005.

[Bonato 05] A. Bonato. "A Survey of Web Graph Models." In Combinatorial and Algorithmic Aspects of Networking: First Workshop on Combinatorial and Algorithmic Aspects of Networking, CAAN 2004, Banff, Alberta, Canada, August 5-7, 2004, Revised Selected Papers, edited by A. López-Ortiz and A. Hamel, pp. 159-172, Lecture Notes in Computer Science 3405. Berlin: Springer, 2005.

[Broder et al. 00] A. Broder, R. Kumar, F. Maghoul, P. Rahaghavan, S. Rajagopalan, R. State, A. Tomkins, and J. Wiener. "Graph Structure in the Web." In Proceedings of the Ninth International World Wide Web Conference, pp. 309-320. Reston, VA: Foretec Seminars, Inc., 2000.

[Chung and Lu 04] F. Chung and L. Lu. "Coupling Online and Offline Analyses for Random Power Law Graphs." Internet Mathematics 1:4 (2004), 409-461.

[Janson et al. 00] S. Janson, T. Łuczak, and A. Ruciński. Random Graphs. New York: Wiley, 2000.

[Euczak and Prałat 06] T. Euczak and P. Prałat. "Protean Graphs." To appear in Internet Mathematics, 2006.

[Pittel et al. 96] B. Pittel, J. Spencer, and N. Wormald. "Sudden Emergence of a Giant k-Core in a Random Graph." J. Combinatorial Theory, Series B 67 (1996), 111151.

[Prałat 06] P. Prałat. "Protean Graphs-Giant Component and its Diameter." Submitted to Discrete Mathematicae, 2006.

[Wormald 99] N. Wormald. "The Differential Equation Method for Random Graph Processes and Greedy Algorithms." In Lectures on Approximation and Randomized Algorithms, edited by M. Karoński and H. J. Prömel, pp. 73-155. Warsaw: PWN, 1999.

[Wormald 04] N. C. Wormald. "Random Graphs and Asymptotics." Section 8.2 in Handbook of Graph Theory, edited by J. L. Gross and J. Yellen, pp. 817-836. Boca Raton: CRC, 2004.

Paweł Prałat, Department of Combinatorics and Optimization, University of Waterloo, Waterloo ON, Canada N2L 3G1 (ppralat@math.uwaterloo.ca)

Nicholas Wormald, Department of Combinatorics and Optimization, University of Waterloo, Waterloo ON, Canada N2L 3G1 (nwormald@math.uwaterloo.ca)

Received November 30, 2005; accepted May 19, 2006. 